\title{
Unravelling Photoinduced Interlayer Spin Transfer Dynamics in 2D Nonmagnetic-Ferromagnetic van der Waals Heterostructures
}

\author{
Junjie He, ${ }^{1,2 *}$ Shuo $\mathrm{Li}^{2}$, Arkamita Bandyopadhyay, ${ }^{1}$ and Thomas Frauenheim ${ }^{1,3^{*}}$ \\ ${ }^{1}$ Bremen Center for Computational Materials Science, University of Bremen, Am Fallturm 1, 2835, \\ Bremen, Germany \\ ${ }^{2}$ Department of Physical and Macromolecular Chemistry \& Charles University Centre of Advanced \\ Materials, Faculty of Science, Charles University in Prague, Hlavova 8, Prague 2, 128 43, Czech \\ Republic \\ ${ }^{3}$ Beijing Computational Science Research Center (CSRC), Beijing 100193 and Shenzhen Computational \\ Science and Applied Research (CSAR) Institute, Shenzhen 518110, China \\ E-mail: junjie.he.phy@gmail.com; thomas.frauenheim@bccms.uni-bremen.de;
}




\section{Methods and computational details}

NM-FGT heterostructures were optimized and their magnetic ground states and band structures were assessed using the Vienna $a b$ initio simulation package (VASP) ${ }^{1,2}$ within the generalized gradient approximation and the Perdew-Burke-Ernzerhof (PBE) exchange-correlation functional. ${ }^{3}$ The projectoraugmented wave method was used to treat the ion-electron interaction. The DFT-D2 method of Grimme was considered for van der Waals corrections. ${ }^{4}$ The plane-wave kinetic-energy cutoff was set to $500 \mathrm{eV}$. The criteria of energy and atom force convergence were set to $10^{-5} \mathrm{eV}$ and $0.01 \mathrm{eV} / \AA$, respectively, using a plane-wave kinetic energy cutoff of $500 \mathrm{eV}$ and adopting a vacuum space of $15 \AA$. The Brillouin zone (BZ) was sampled using $11 \times 11 \times 1$ Gamma-centered Monkhorst-Pack grids to calculate relaxation and electronic structures.

To identify the spin dynamics of FGT-based heterostructures under the influence of ultrafast laser pulses, we performed real-time time-dependent density functional theory (rt-TDDFT) calculations. The time evolving state functions $(\psi)$ were calculated by solving the time dependent Kohn-Sham (KS)

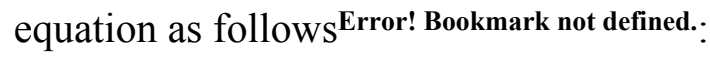

$i \frac{\partial \psi_{j}(\mathbf{r}, t)}{\partial t}=\left[\frac{1}{2}\left(-i \nabla+\frac{1}{c} \mathbf{A}_{\mathbf{e x t}}(t)\right)^{2}+v_{s}(\mathbf{r}, t)+\frac{1}{2 c} \sigma \cdot \mathbf{B}_{\mathbf{s}}(\mathbf{r}, t)+\frac{1}{4 c^{2}} \sigma \cdot\left(\nabla v_{s}(\mathbf{r}, t) \times-i \nabla\right)\right] \psi_{j}(\mathbf{r}, t)$

where $\mathbf{A}_{\text {ext }}(t)$ and $\sigma$ represent vector potential and Pauli matrices. The KS effective potential $v_{S}(\mathbf{r}, t)=v_{\text {ext }}$ $(\mathbf{r}, t)+v_{H}(\mathbf{r}, t)+v_{x c}(\mathbf{r}, t)$ can be decomposed into the external potential $v_{\text {ext }}$, the classical Hartree potential $v_{H}$, and the exchange-correlation $(\mathrm{XC})$ potential $v_{x c}$, respectively. The KS magnetic field can be written as $\mathbf{B}_{\mathbf{s}}(\mathbf{r}, t)=\mathbf{B}_{\mathbf{e x t}}(\mathbf{r}, t)+\mathbf{B}_{\mathbf{x c}}(\mathbf{r}, t)$, where $\mathbf{B}_{\mathbf{e x t}}$ and $\mathbf{B}_{\mathbf{x c}}$, which may represent the magnetic field of the applied laser pulse plus an additional magnetic field and XC magnetic field, respectively. The last term in Eq. (1) stands for SOC. We kept the nuclei of system fixed during the simulation.

The spin-dynamics of NM/FGT heterostructures is governed by the flow of the spin current, which can 
be described by spin current density tensor, $\mathbf{J}(\mathbf{r})$. The equation of motion of the magnetization density with respect to the spin current density tensor can be defined as:

$$
\frac{\partial \mathbf{m}(\mathbf{r})}{\partial t}=-\nabla \cdot \mathbf{J}(\mathbf{r})-\frac{1}{c} \mathbf{m}(\mathbf{r}) \times \mathbf{B}_{s}(\mathbf{r})
$$

Photoinduced dynamics calculations were made using a fully non-collinear version of rt-TDDFT and a full-potential augmented plane-wave ELK code. ${ }^{5}$ A regular mesh in a k-space of $7 \times 7 \times 1$, a smearing width of $0.027 \mathrm{eV}$, and a time step of $\Delta t=0.1$ a.u. were used to simulate excited dynamics. The laser pulses that were used in the present study were linearly polarized (in-plane polarization) at a selected frequency. All calculations were performed using adiabatic local spin density approximations (ALSDA). ${ }^{6}$
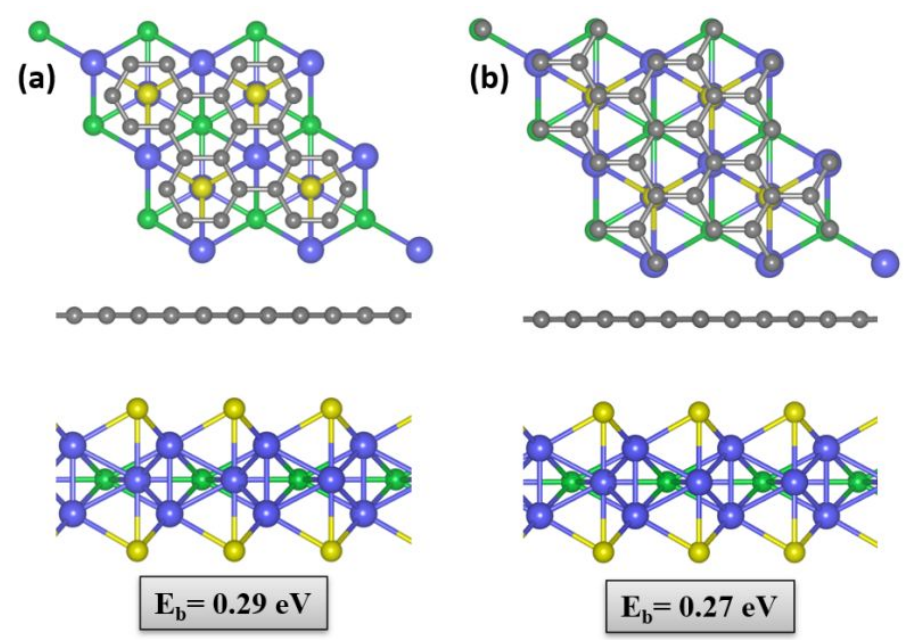

Figure S1: Top and side view of stacking configurations in models 1 (a) and 2 (b) of graphene-FGT heterostructures. $\mathrm{E}_{\mathrm{b}}$ is defined as interlayer interactions between graphene and FGT layer. Blue, green, yellow and grey spheres represent Fe, Ge, Te and C atoms, respectively. 
(a)
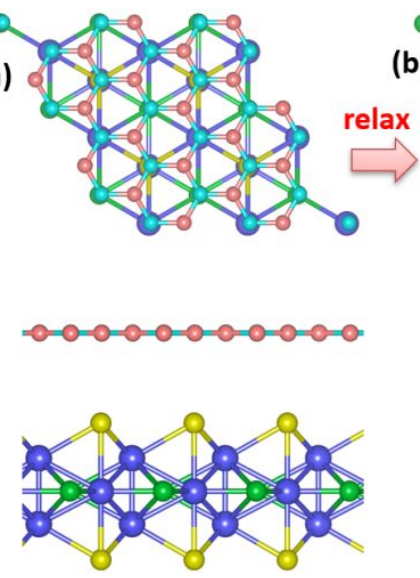

(b)

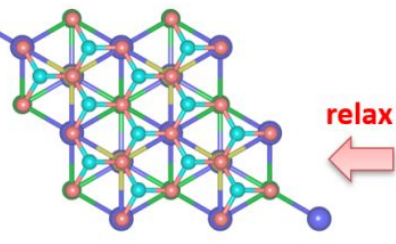

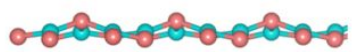

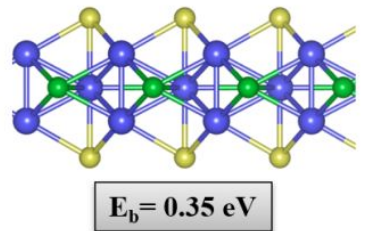

(c)
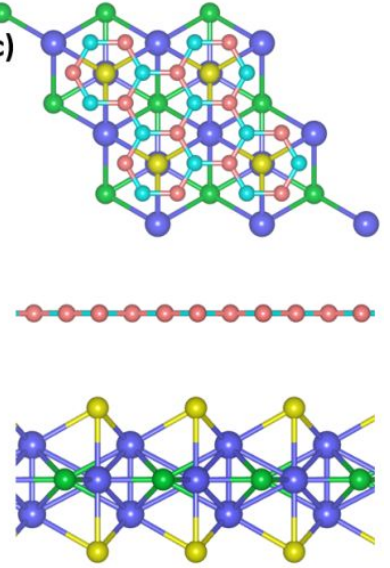

Figure S2: Top and side views of stacking configurations in models 1(a), 2 (b) and 3 (c) of $h$-BN-FGT heterostructures. In models 1 and 3, the initial stacking configurations are not stable. These configurations relax to model 2 during the optimized process. $\mathrm{E}_{\mathrm{b}}$ is defined as interlayer interactions between $h$ - $\mathrm{BN}$ and FGT layers. Blue, green, yellow, peach and aqua spheres represent $\mathrm{Fe}, \mathrm{Ge}, \mathrm{Te}, \mathrm{B}$ and $\mathrm{N}$ atoms, respectively.

(a)
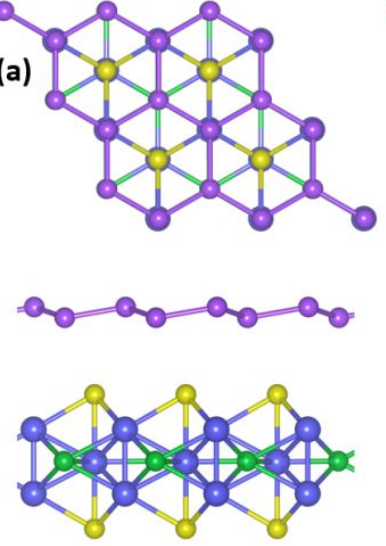

$E_{b}=0.34 \mathrm{eV}$
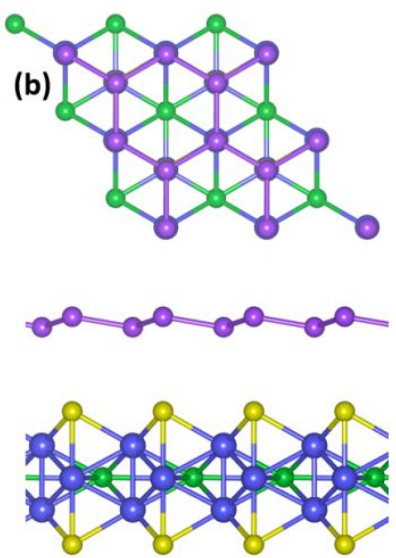

$E_{b}=0.25 \mathrm{eV}$
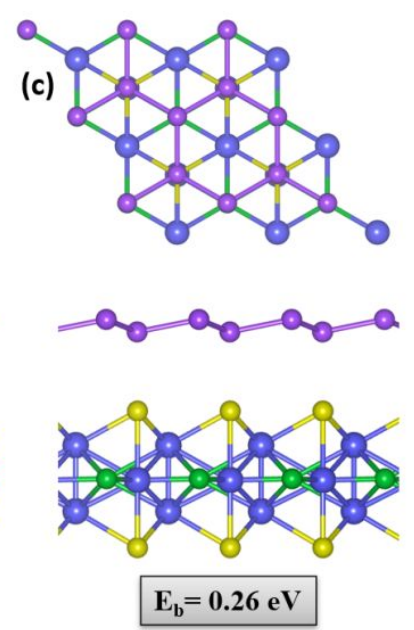
Figure S3: Top and side views of stacking configurations in models 1 (a), 2 (b) and 3 (c) of silicene-FGT heterostructures. $\mathrm{E}_{\mathrm{b}}$ is defined as interlayer interactions between silicene and FGT layer. Blue, green, yellow and purple spheres represent $\mathrm{Fe}, \mathrm{Ge}, \mathrm{Te}$ and $\mathrm{Si}$ atoms, respectively.
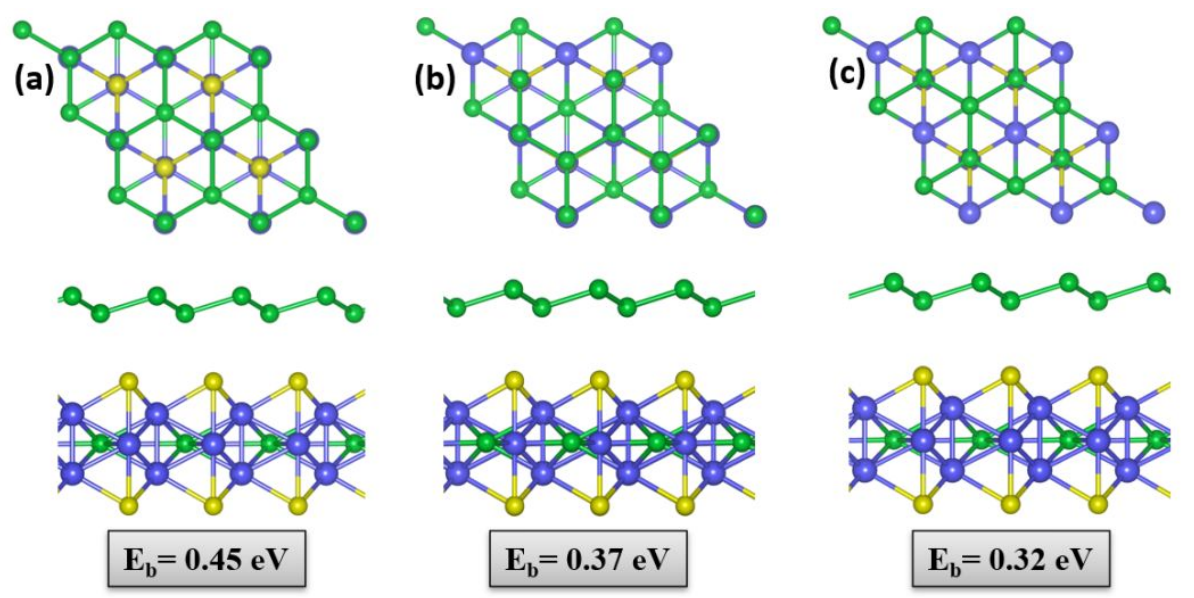

Figure S4: Top and side views of stacking configurations in models 1 (a), 2 (b) and 3 (c) of germaneneFGT heterostructures. $\mathrm{E}_{\mathrm{b}}$ is defined as interlayer interactions between germanene and FGT layers. Blue, green, yellow and grey spheres represent $\mathrm{Fe}, \mathrm{Ge}$, and Te atoms, respectively.

(a)
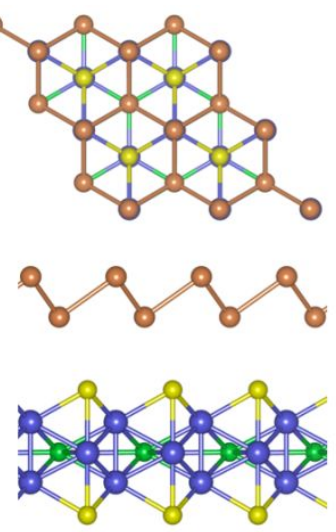

$$
E_{b}=0.43 \mathrm{eV}
$$
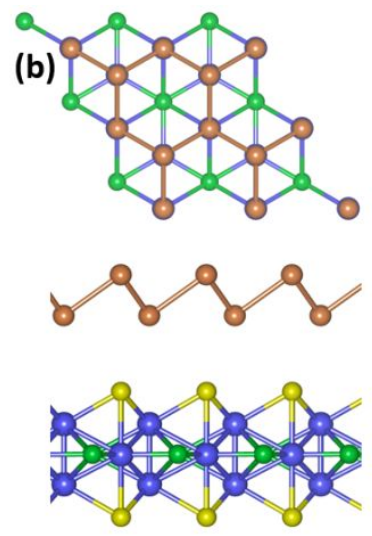

$$
E_{b}=0.40 \mathrm{eV}
$$
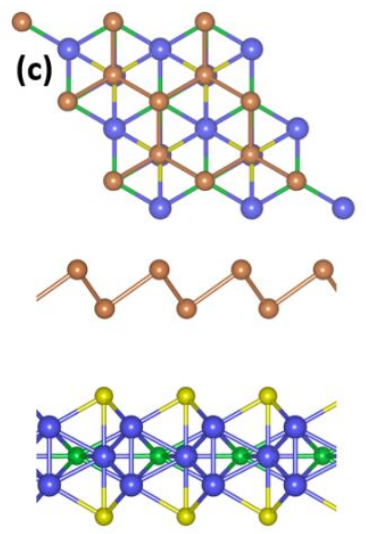

$E_{b}=0.24 \mathrm{eV}$ 
Figure S5. Top and side views of stacking configurations in models 1 (a), 2 (b) and 3 (c) of antimoneneFGT heterostructures. $\mathrm{E}_{\mathrm{b}}$ is defined as interlayer interactions between antimonene and FGT layers. Blue, green, yellow and brown spheres represent $\mathrm{Fe}, \mathrm{Ge}, \mathrm{Te}$ and $\mathrm{Sb}$ atoms, respectively.

Table S1. The mismatch of Lattice $(M L)$ defined $M L=\left(L_{N M}-L_{F G T}\right) / L_{F G T}$. The positive and negative values of $L$ represent the ratio between stretch/tensile and compressive forces of NM materials, respectively. $\mathrm{M}_{\mathrm{NM} 1}, \mathrm{M}_{\mathrm{NM} 2}, \mathrm{M}_{\mathrm{Fe} 1}, \mathrm{M}_{\mathrm{Fe} 2}, \mathrm{M}_{\mathrm{Fe} 3}$ stand for the local magnetic moment of NM and FGT, as shown in Figure 1. $d$ is the distance between NM and FGT layers.

\begin{tabular}{cccccccc}
\hline Structure & $\mathrm{ML}$ & $\mathrm{M}_{\mathrm{NM} 1}\left(\mu_{B}\right)$ & $\mathrm{M}_{\mathrm{NM} 2}\left(\mu_{B}\right)$ & $\mathrm{M}_{\mathrm{Fe} 1}\left(\mu_{B}\right)$ & $\mathrm{M}_{\mathrm{Fe} 2}\left(\mu_{B}\right)$ & $\mathrm{M}_{\mathrm{Fe} 3}\left(\mu_{B}\right)$ & $\mathrm{d}(\AA)$ \\
\hline graphene/FGT & $5.53 \%$ & 0.001 & 0.001 & 2.474 & 2.450 & 1.422 & 3.373 \\
$h$-BN/FGT & $7.38 \%$ & -0.001 & 0.0 & 2.471 & 2.442 & 1.434 & 3.110 \\
silicene/FGT & $-4.49 \%$ & -0.019 & 0.034 & 2.448 & 2.447 & 1.409 & 2.948 \\
germanese/FGT & $1.73 \%$ & -0.007 & 0.027 & 2.462 & 2.406 & 1.392 & 2.834 \\
antimonene/FGT & $1.61 \%$ & -0.001 & 0.002 & 2.447 & 2.436 & 1.495 & 2.997 \\
\hline
\end{tabular}



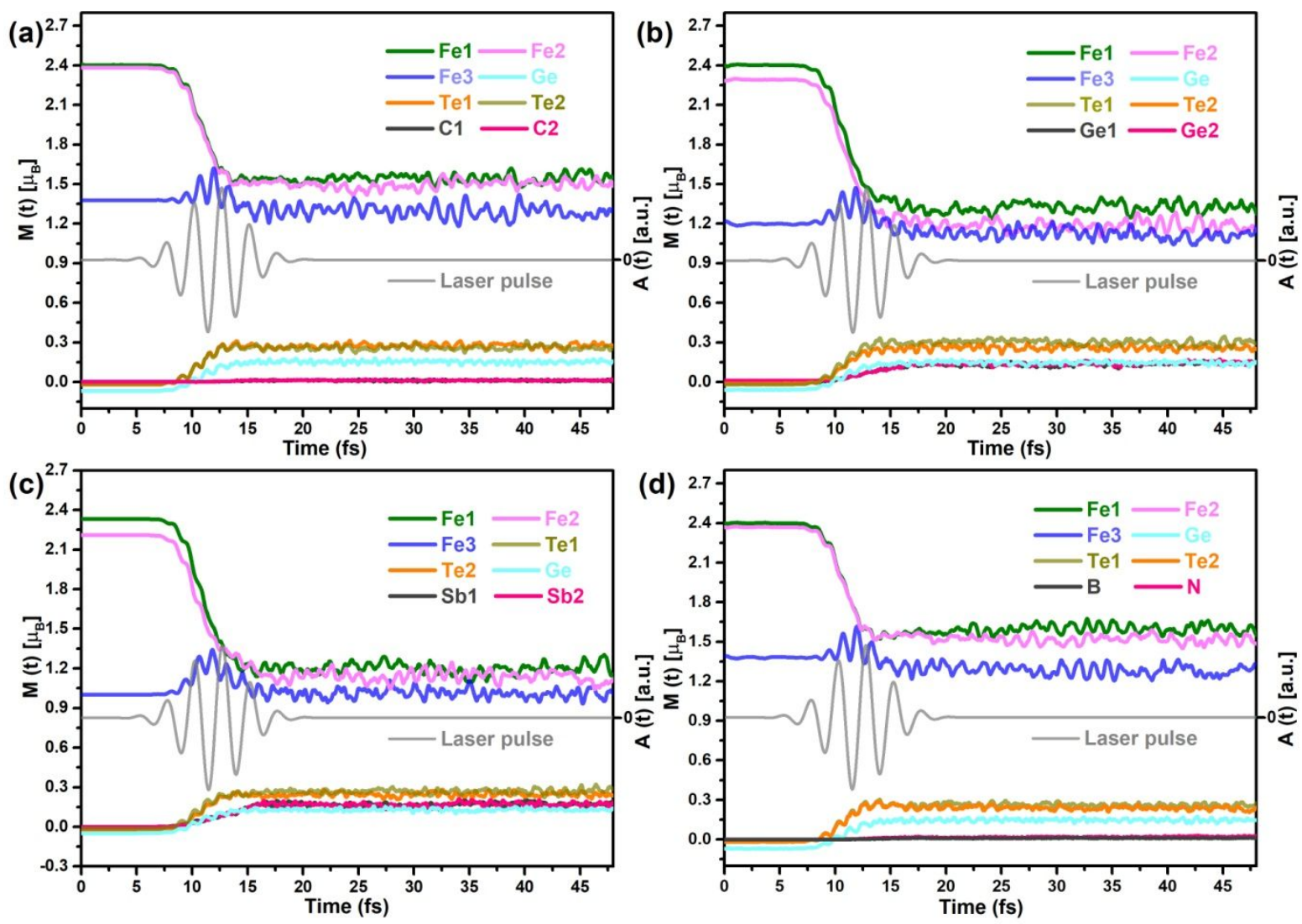

Figure S6: Ultrafast laser-induced local magnetic moment dynamics of graphene-FGT(a), germaneneFGT (b), antimonene-FGT (c) and $h$-BN-FGT (d). The $\mathbf{A}(\mathrm{t})$ of the laser pulse is also shown.
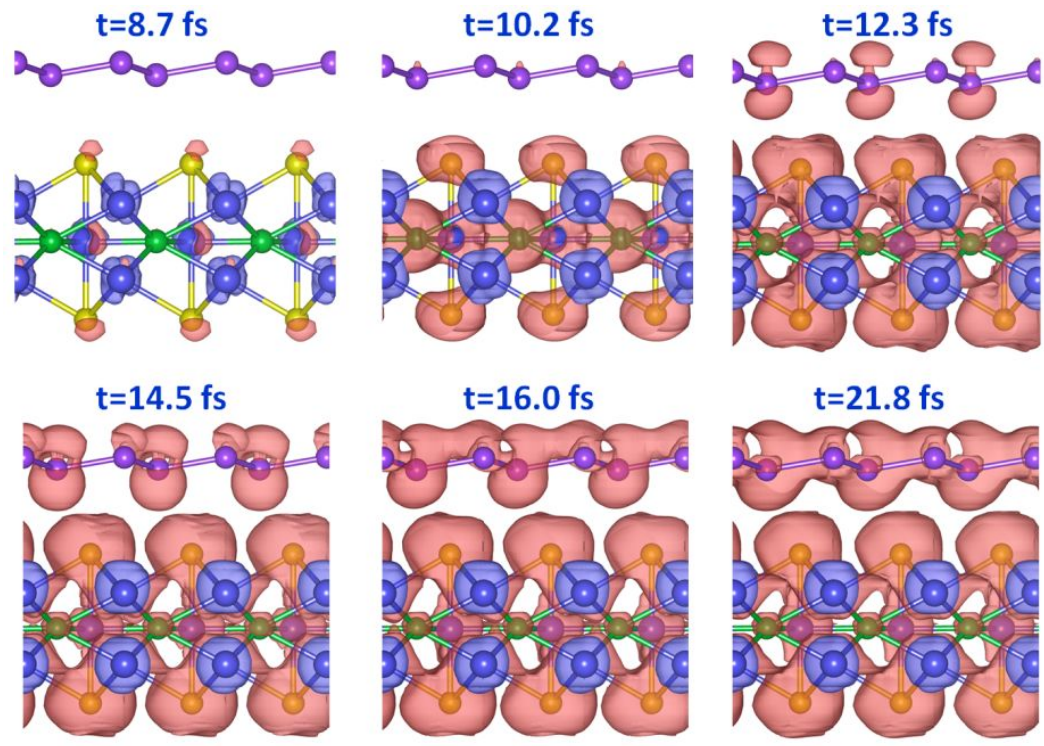

Figure S7: Snapshots of changes $(\Delta \mathrm{M}(\mathrm{t})=\mathrm{M}(\mathrm{t})-\mathrm{M}(0))$ in the magnetization density of silicene-FGT at different time point.

Red (blue) domains of the iso-surfaces indicate electron spin up (down). The iso-surfaces are depicted with a cutoff value of $0.002 \mathrm{e} / \AA^{3}$. 

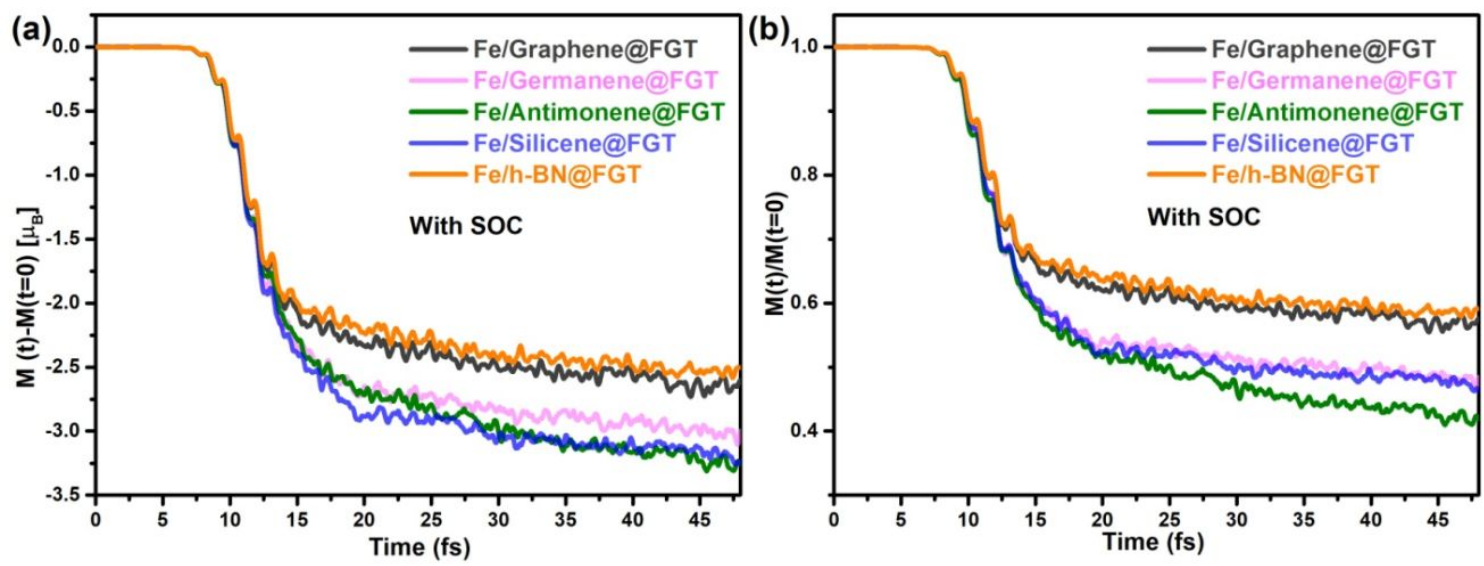

Figure S8. Change in magnetic moment $(\Delta \mathrm{M}(\mathrm{t})=\mathrm{M}(\mathrm{t})-\mathrm{M}(0))$ (a) and normalized moment $(\mathrm{mM}(\mathrm{t}) / \mathrm{M}(0))$ dynamics (b) as a function of time (in fs) with SOC.
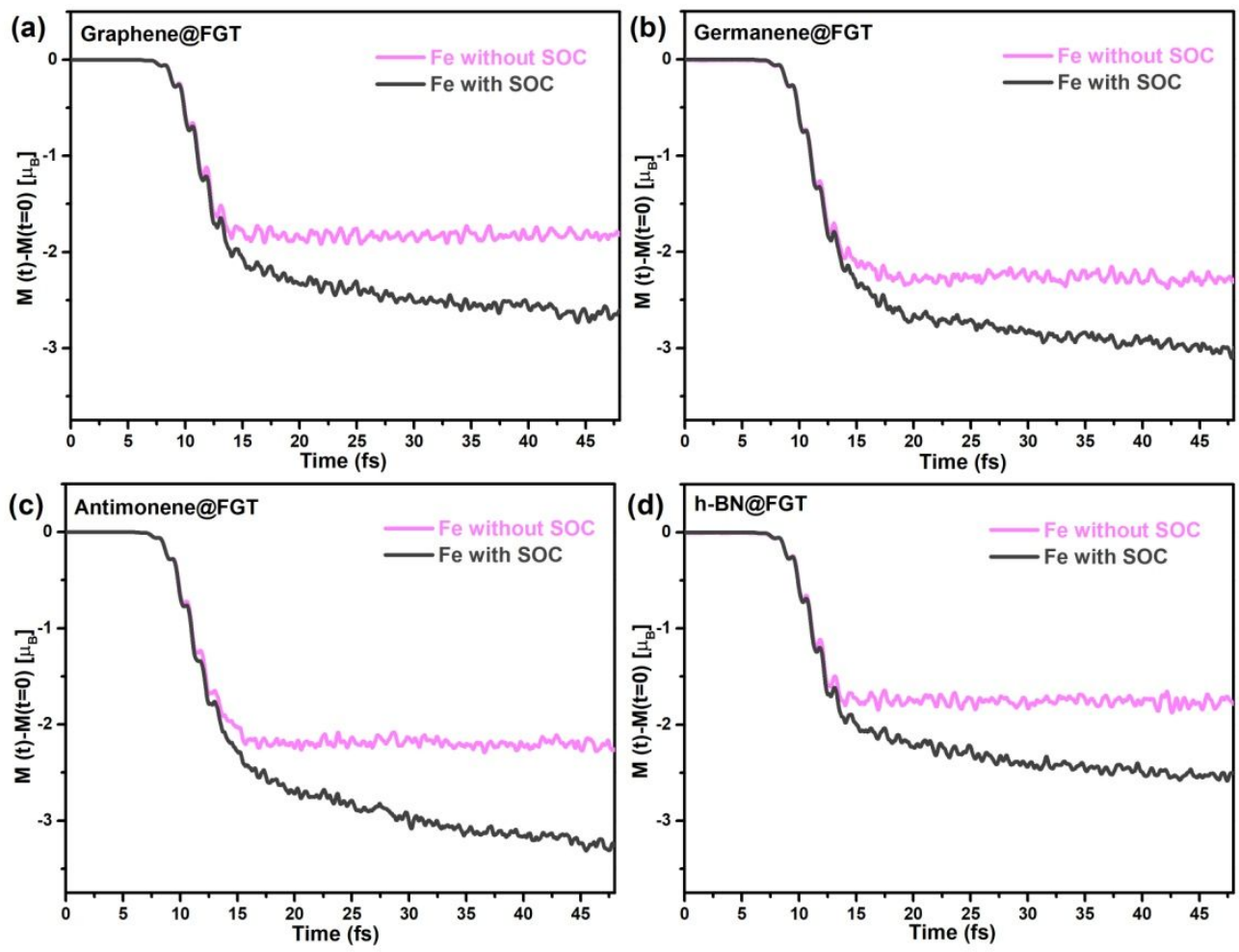

Figure S9: Changes in the moment, $\Delta \mathrm{M}(\mathrm{t})=\mathrm{M}(\mathrm{t})-\mathrm{M}(0)$, of graphene-FGT (a), germanene-FGT (b), antimonene-FGT (c), and $h$-BN-FGT (d) heterostructures as a function of time (in fs) calculated with (black lines) and without SOC (pink lines) 

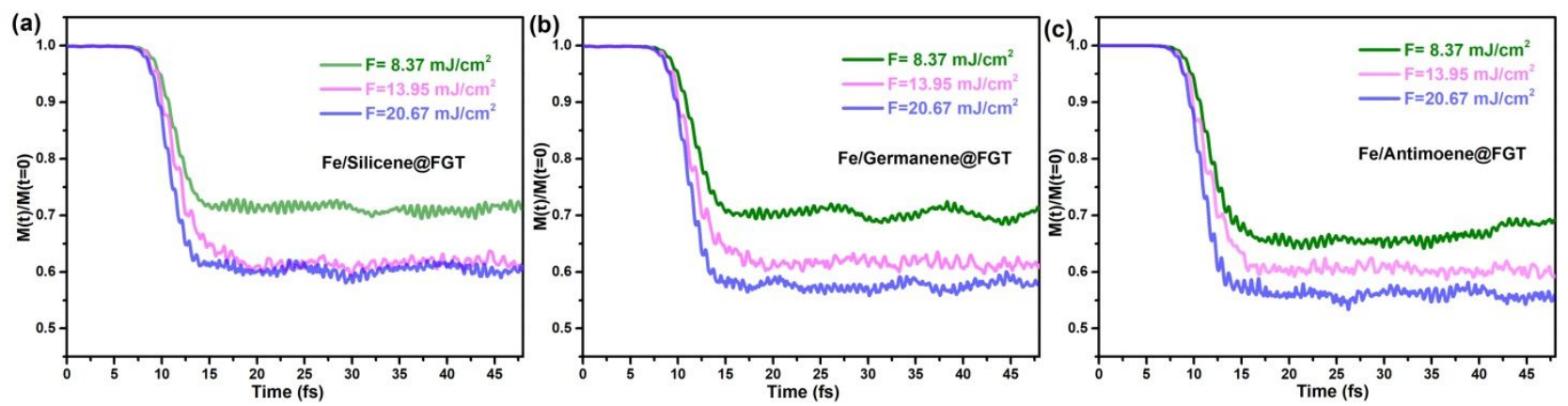

Figure S10: Normalized moment $(\mathrm{M}(\mathrm{t}) / \mathrm{M}(0))$ dynamics as a function of time (in $\mathrm{fs}$ ) at various fluence (F) rates of Fe elements in silicene-FGT (a), germanene-FGT (b), and antimonene-FGT (c), respectively
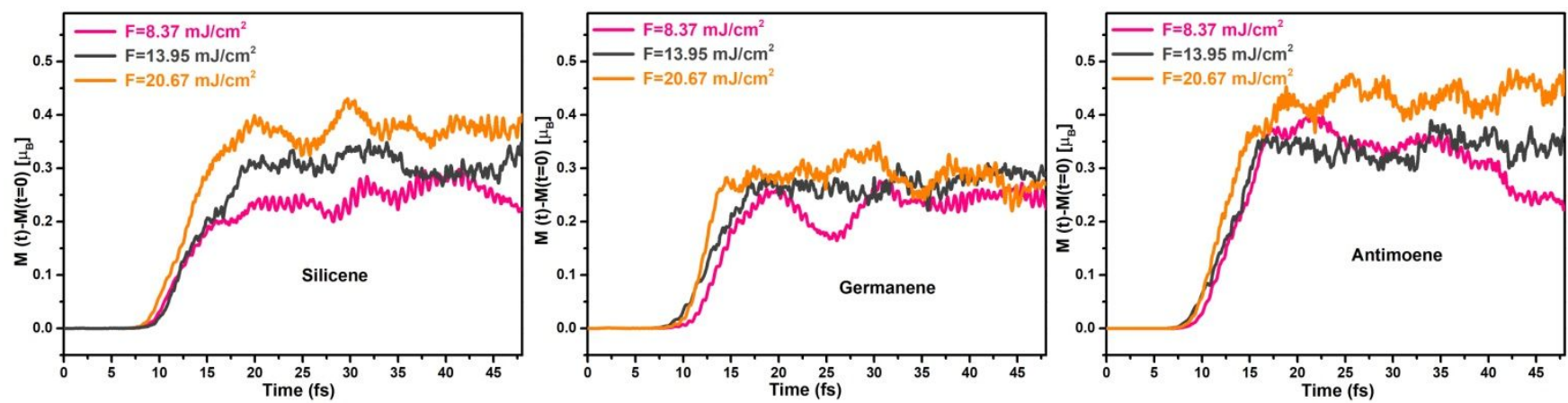

Figure S11: Changes in moment $(\Delta \mathrm{M}(\mathrm{t})=\mathrm{M}(\mathrm{t})-\mathrm{M}(0))$ dynamics as a function of time (in $\mathrm{fs}$ ) at various Fluence (F) rates of NM layers in silicene-FGT (a), germanene-FGT (b), and antimonene-FGT (c), respectively

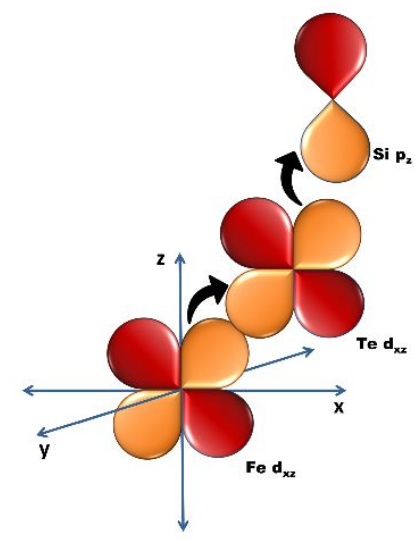

Figure S12: Orbital diagram for Fe- $d_{\mathrm{xz}}, \mathrm{Te}-d_{\mathrm{xz}}$ and $\mathrm{Si}-p_{\mathrm{z}}$ states are shown. The spindependent charge transfer pathway also marked, respectively. 


\section{Reference}

(1) Kresse, G.; Jürgen, H. Ab initio molecular dynamics for liquid metals. Phys. Rev. B 1993, 47, 558.

(2) Kresse, G.; Joubert, D. From ultrasoft pseudopotentials to the projector augmented-wave method, Phys. Rev. B 1999, 59, 1758.

(3) Perdew, J. P.; Burke, K.; Ernzerhof, M. Generalized Gradient Approximation Made Simple. Phys. Rev. Lett. 1996, 77, 3865.

(4) Grimme, S. Semiempirical GGA-type density functional constructed with a long-range dispersion correction. J. Comput. Chem. 2006, 27(15), pp.1787-1799.

(5) Dewhurst, J. K.; Sharma, S.; et al. Elk code, elk.sourceforge.net (accessed Oct 1, 2020).

(6) von Barth, U.; Hedin, L. A local exchange-correlation potential for the spin polarized case, J. Phys. C: Solid State Phys. 1972, 5, 1629. 